\title{
Dynamic sensitivity analysis of CNC machine tools in static state
}

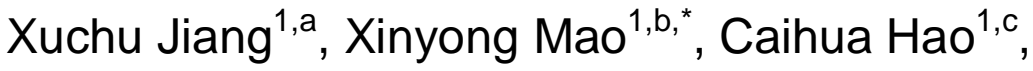 \\ Huanbin $\mathrm{He}^{1, \mathrm{~d}}$, Chao Qin ${ }^{1, \mathrm{e}}$, Bin $\mathrm{Li}^{1, \mathrm{f}}$ \\ ${ }^{1}$ School of Mechanical Science and Engineering, Huazhong University of Science and \\ Technology, Wuhan 430074, China \\ axuchujiang@hust.edu.cn, ${ }^{b}$ maoxyhust@163.com, ${ }^{c} 15997405871 @ 163 . c o m$, \\ dhehuanbin@126.com, ${ }^{\mathrm{e}} 17771788410 @ 163 . c o m,{ }^{\mathrm{f} l i b i n 999 @ h u s t . e d u . c n ~}$
}

Keyword : CNC machine tool, Operational mode, Dynamic sensitivity, Tool wear characterization

\begin{abstract}
This paper mainly discusses the tool condition monitoring based on dynamic sensitivity, and explores a method for characterizing tool condition characterization. Firstly, the sensitive directions and components of different orders low modes were analyzed in the static state. Modal parameters and the dominant mode are also identified. Secondly, dynamic sensitivity analysis of the tool-workpiece system is analyzed by using operational modes. For the dominant mode, the modal sensitivity is high in both static and dynamic state. Although many factors such as boundary condition change a lot in cutting conditions, the modal sensitive parts do not change. It indicates that the modal sensitivity is the basic attribute. Therefore, it is reliable to analyze the dynamic sensitivity of the tool-workpiece system by the amplitude change of the operational modes during the cutting process.
\end{abstract}

\section{Introduction}

Tool condition monitoring is very important for process automation. The excessive tool wear can cause the machining dimension distortion, increase the scrap rate and the production cost [1]. Tool condition monitoring has an important effect on slowing tool wear rate, workpiece surface quality control and process optimization. Tool wear monitoring has two basic methods currently, one is direct monitoring method, the other is indirect monitoring method. Most of the methods are indirect [2-4].

We can use visual or optical signals to analyze the tool state in direct monitoring method. However, it is difficult to be used in the actual machining process. The most widely used monitoring signals in indirect monitoring method are acoustic emission, cutting force and vibration acceleration signals [8]. The biggest limitation is not the sensing technology but the analysis technology [9].

The correlation between the change of cutting force and tool wear has been widely recognized. There are some advantages of using vibration signal to monitor the tool state, such as robustness, high reliability and fast response, which are very important for online real-time monitoring.

The research of using vibration signals to carry on the tool condition monitoring is discussed in this paper. There are mainly four kinds of characteristic signals that characterize the tool wear state: natural frequency amplitude, high frequency band amplitude, tooth frequency amplitude and frequency band energy. 
Lim found that there was a strong correlation between tool wear and natural frequency amplitude of the tool rod [5]. Chelladurai analyzed the correlation between peak amplitude of high frequency band and tool wear [1]. Barreiro analyzed the correlation between maximum amplitude of high frequency band and tool wear [7]. Tomas analyzed the correlation between PSD energy change of tool tooth frequency and tool wear [6].

The present tool wear identification methods have high correlation with the cutting parameters, the application of the identification methods is limited. So the trend of tool condition monitoring is to find a tool wear identification method which is independent of the cutting parameters.

Dimla found that the tool tip wear might better characterize the tool life than the wear of tool side rack [1]. So we will mainly study the correlation between tool tip wear and vibration signals of turning tool. Change factor of tool-workpiece system in cutting process can lead to the change of low frequency operational mode of CNC machine tool structure. So we will study tool wear of turning process based on operational modal analysis (OMA) in this paper.

The dynamic change of the tool-workpiece system in cutting process is studied based on OMA. A frequency domain method named op.polymax algorithm can be used to process the PSD of vibration acceleration response signals. Each order mode of CNC machine tool can be accurately determined in machining process. We analyze the change factor sensitivity of tool-workpiece system in different directions as well as each order low-frequency operational mode of different parts in continuous cutting process. This is defined as the dynamic sensitivity method. Dynamic sensitivity changes with the cutting excitation energy. And the corresponding direction's structure dynamic sensitivity parameters will change significantly. Thus we can obtain the variation of wear. The method is significantly different from the traditional spectrum analysis.

In this paper, we will use dynamic sensitivity method to study the tool condition monitoring.

\section{Experimental design of dynamic sensitivity method}

Firstly, a common experimental system of impact and cutting experiments is shown in Fig.1.

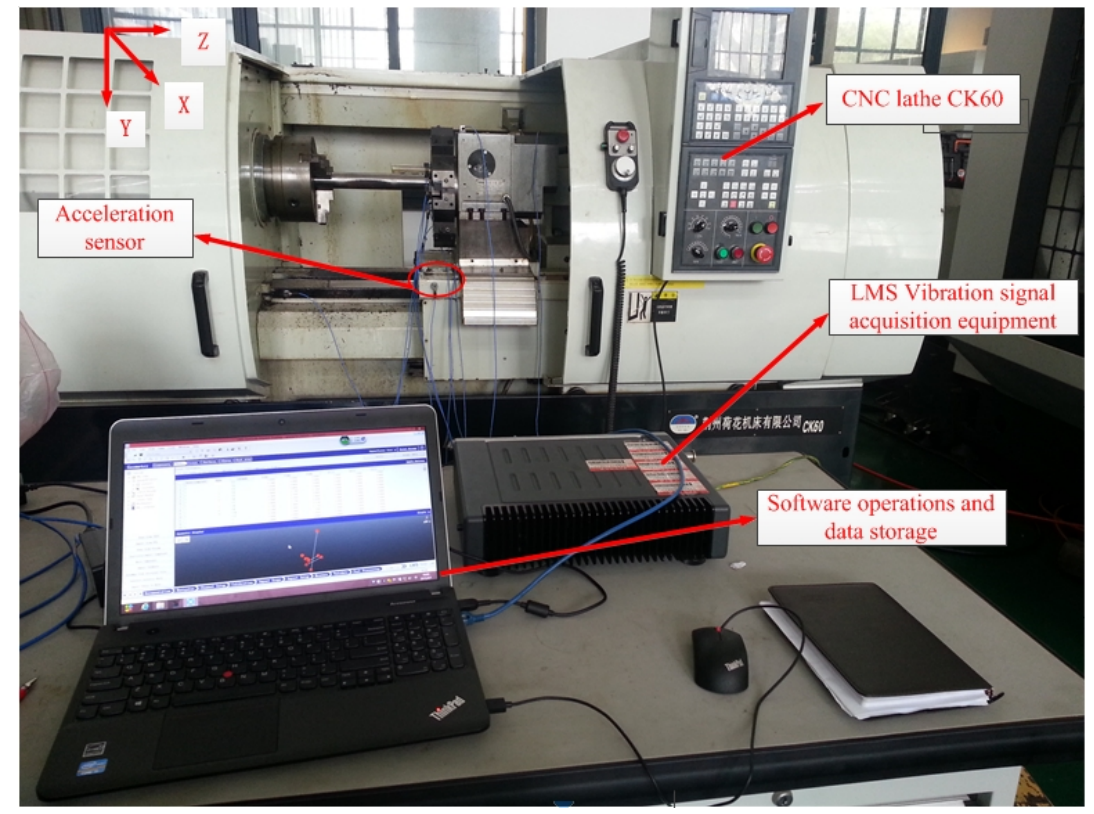

Fig. 1 Experimental system

The details of the experimental system are shown in table 1. 
Table 1 Details of the experimental system

\begin{tabular}{|l|l|}
\hline Components & Details \\
\hline Machine Tool & CNC lathe K60 \\
\hline $\begin{array}{c}\text { Data acquisition and } \\
\text { analysis system }\end{array}$ & LMS SCADAS Mobile SCM05 \& LMS Test. Lab 10B \\
\hline Cutting tool & Mitsubishi turning tools,type:PTGNR2525M16 \\
\hline Workpiece & 45 steel \\
\hline $\begin{array}{l}\text { Vibration response } \\
\text { sensor }\end{array}$ & PCB-356A15, the measurement frequency band is 5000Hz \\
\hline
\end{tabular}

Secondly, cutting a large-diameter workpiece is taken as an example to detail the cutting process system and arrangement of sensor measuring points as shown in Fig.2.

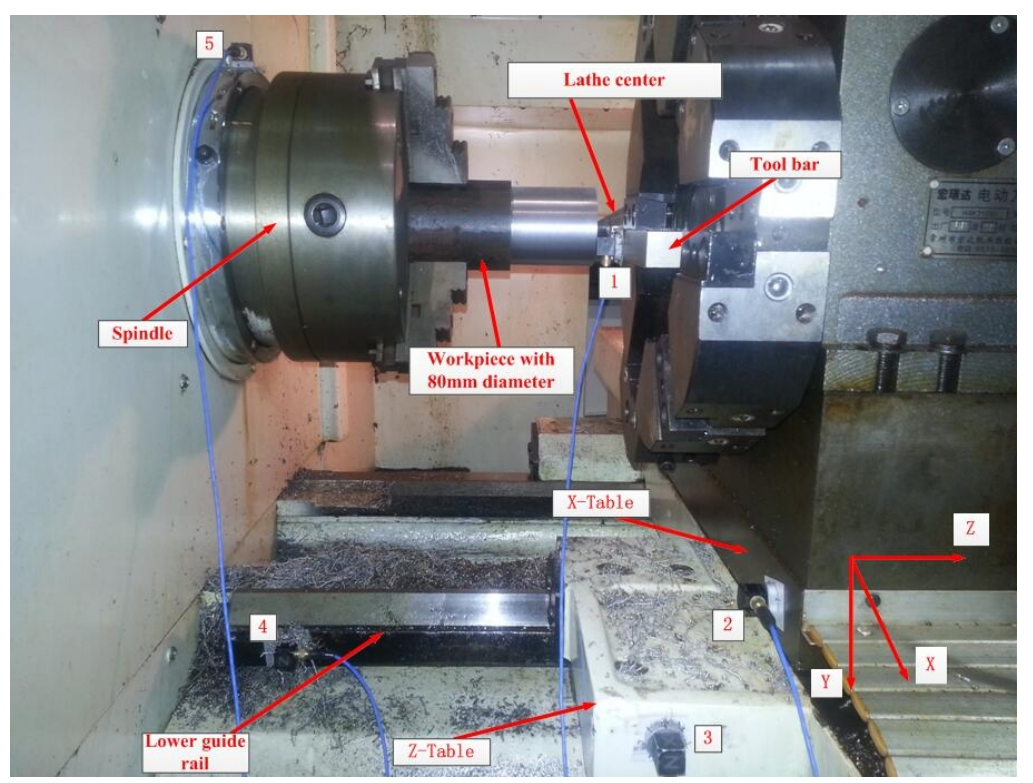

Fig. 2 Schematic diagram of Process system and sensor measuring points

The measuring point 1 is on the tool rod near the cutting edge. The measuring point 2 is on the $\mathrm{X}$ table. The measuring point 3 is on the $\mathrm{Z}$ table. The measuring point 4 is on the down guide and the measuring point 5 is on the spindle side.

\section{Dynamic sensitivity analysis in static state}

This section mainly analyzes the dynamic sensitivity in static state. The sensitive directions, sensitive components and dominant modes of each low frequency mode in $0-120 \mathrm{~Hz}$ were analyzed.

The low frequency modal sensitivity analysis in static state utilized the impact experiment data. We impacted the $\mathrm{Z}$ direction of the tool, $\mathrm{Z}$ direction of $\mathrm{X}$ table, $\mathrm{X}, \mathrm{Y}, \mathrm{Z}$ direction of $\mathrm{Z}$ table in the impact experiments, then obtained the FRF. Fig. 3 shows the impact test in $+Z$ direction of $X$ table. 


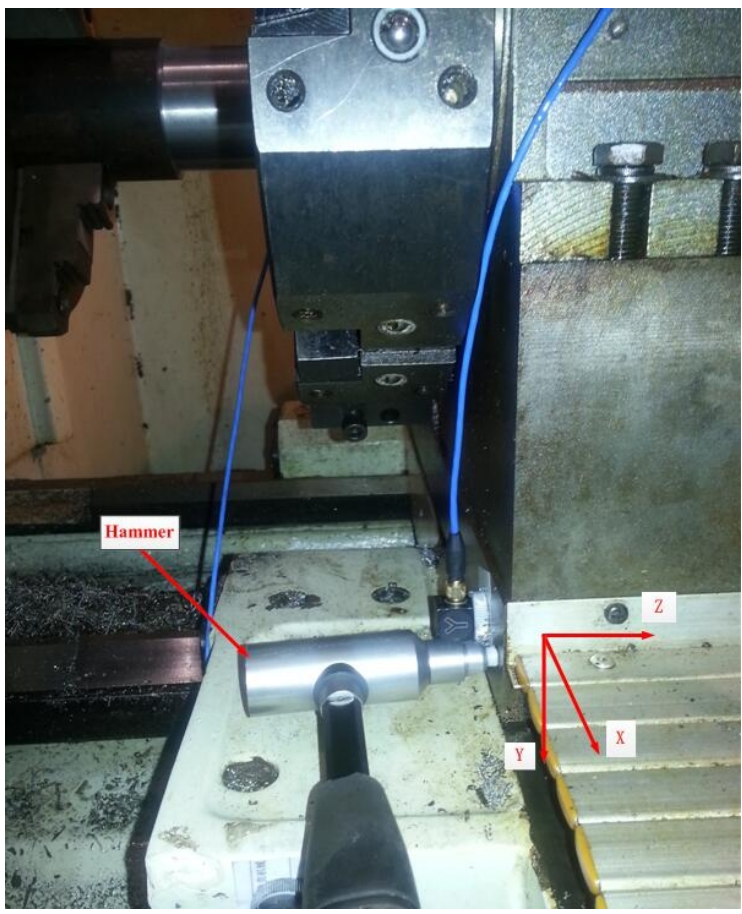

Fig. 3 Schematic diagram of impact test

The frequency response function (FRF) of each measuring point were obtained after the impact test, and then the steady-state diagram calculated by PolyMAX algorithm is shown in Fig.4.

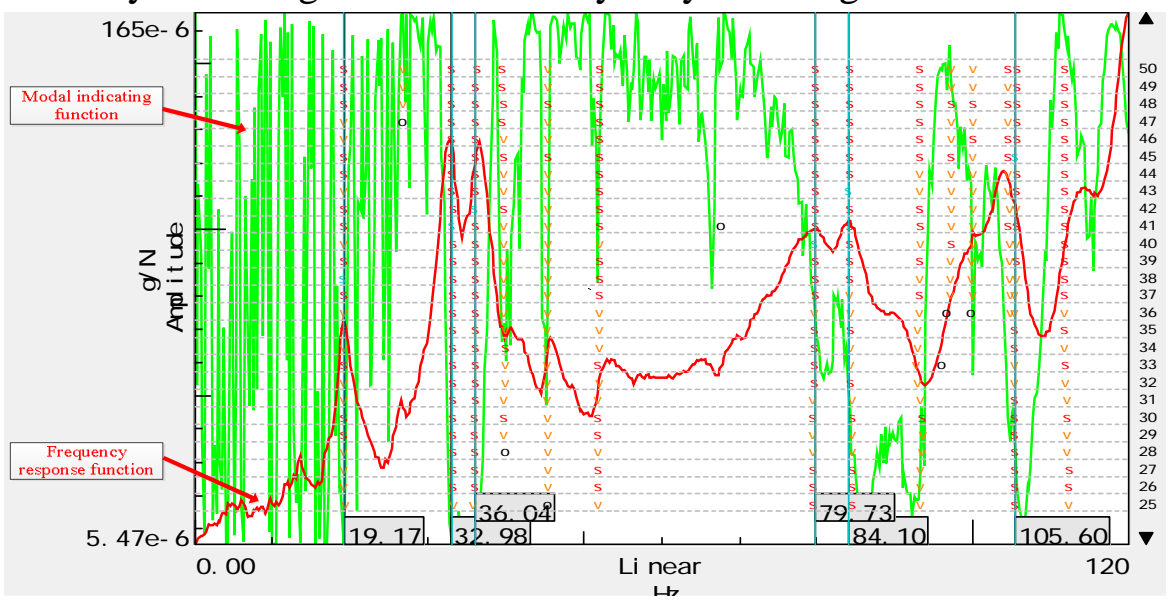

Fig. 4 The steady - state diagram of the modes in FRF

In Fig.4, the left ordinate represents the amplitude and the right ordinate represents the calculated model order. The red curve in the figure represents the calculated FRF. The green curve in the figure represents the modal indicating function. When the modal indicating function is close to zero, it means that the corresponding frequency is likely to be an order mode.

\section{Dynamic sensitivity analysis of the low frequency mode in static state.}

The sensitivity analysis is conducted mainly from the sensitivity directions, the sensitivity parts and the dominant mode. The principle of judgment is: According to the comprehensive analysis of the FRF of different measuring points, if the mode peak exists in the certain direction regardless of different measuring points, and the amplitude is relatively large, then the mode is sensitive to this direction. With the same impact, the component with the largest FRF amplitude is considered as the sensitive part. According to this principle, the dominant mode can be determined. 


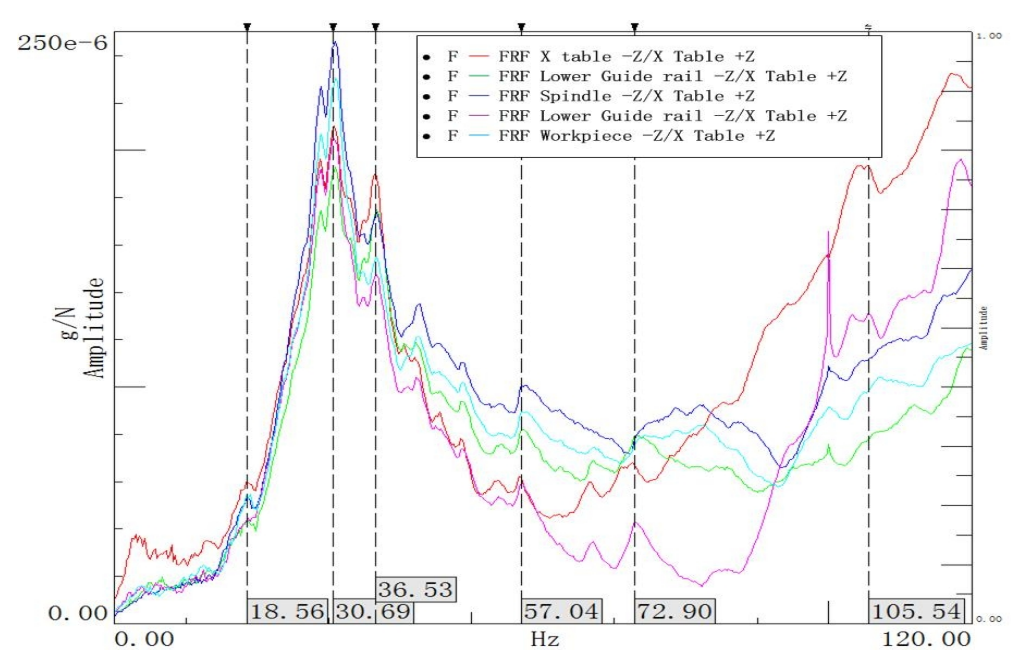

Fig. 5 FRF of the measuring points when impacting $\mathrm{Z}$ direction of $\mathrm{X}$ table

Fig. 5 shows that the amplitudes of $30 \mathrm{~Hz}$ and $36 \mathrm{~Hz}$ are significant, so they are both sensitive to the $\mathrm{Z}$ direction. The amplitude of $30 \mathrm{~Hz}$ is the largest at the spindle measuring point, so it is sensitive to the measuring point on the spindle. The amplitude of the $36 \mathrm{~Hz}$ is the largest at the impact point located on $\mathrm{X}$ table and the amplitude is the second on the spindle away from the impact point. So the $36 \mathrm{~Hz}$ is also sensitive to the spindle. This is an interaction that takes the three-dimensional excitation of the tool tip, the structural vibration complexity as well as the transfer function between the various measuring points into account. The aim is to determine the sensitive modes of the different components.

\section{Modal Parameters and Sensitivity Summarization in Low Frequency Band of CNC Machine Tool}

By analyzing the frequency steady-state diagram of each direction and each measuring point in the five impact experiments and combining with the sensitive components as well as sensitive directions of each mode, the results are summarized in Table 2.

Table 29 orders modes and sensitivity in $0-120 \mathrm{~Hz}$ frequency band of the CNC lathe

\begin{tabular}{|c|c|c|c|c|}
\hline $\begin{array}{l}\text { Mode parameters } \\
\text { Order }\end{array}$ & $\begin{array}{l}\text { Natural Frequency } \\
(\mathrm{Hz})\end{array}$ & $\begin{array}{l}\text { Damping } \\
\text { ratio ( } \%)\end{array}$ & $\begin{array}{l}\text { Sensitive } \\
\text { direction }\end{array}$ & $\begin{array}{l}\text { Sensitive } \\
\text { components }\end{array}$ \\
\hline First order & 19.045 & 1.49 & $X$ & spindle \\
\hline Second order & 30.769 & 1.14 & $\mathrm{Z}$ & spindle \\
\hline Third order & 36.218 & 0.99 & $\mathrm{Z}$ & spindle \\
\hline Forth order & 77.391 & 1.85 & & \\
\hline Fifth order & 85.550 & 3.85 & $X$ & tool \\
\hline Sixth order & 89.579 & 0.39 & $X$ & tool \\
\hline Seventh order & 105.149 & 0.41 & $\mathrm{X}$ & spindle \\
\hline
\end{tabular}

Table 2 shows that $35 \mathrm{~Hz}$ is the dominant mode of the spindle in $\mathrm{Z}$ direction, while $85 \mathrm{~Hz}$ is the dominant mode of the tool in $\mathrm{X}$ direction and $19 \mathrm{~Hz}$ is the dominant mode of the spindle in $\mathrm{X}$ direction. The dominant mode in $\mathrm{X}$ direction is $85 \mathrm{~Hz}$, and the dominant mode in $\mathrm{Z}$ direction is 36 $\mathrm{Hz}$. 


\section{Conclusion}

In static state, $85 \mathrm{~Hz}$ of $\mathrm{X}$ direction is the most sensitive to the tool. In $0-120 \mathrm{~Hz}$ frequency band, the modal sensitive directions in the lower frequency bands $30-40 \mathrm{~Hz}$ are mainly Z-direction of the spindle. The modal sensitive directions in the higher frequency bands $80-90 \mathrm{~Hz}$ are $\mathrm{X}$ direction of the tool. And the lowest mode $19 \mathrm{~Hz}$ and the highest mode $105 \mathrm{~Hz}$ are $\mathrm{X}$ direction of the spindle. So the modal sensitivity has a certain regularity.

The dynamic sensitivity analysis under operating conditions was carried out. Because the reasons for space is not detailed. For the dominant mode, the modal sensitivity is high in both static and dynamic state. Although many factors such as boundary condition change a lot in cutting conditions, the modal sensitive parts do not change. It indicates that the modal sensitivity is the basic attribute. Therefore, it is reliable to analyze the dynamic sensitivity of the tool-workpiece system by the amplitude change of the operational modes during the cutting process.

\section{Acknowledgements}

The research is supported by the National Natural Science Foundation of China under Grant No. 51275188 and 51375193, and the Key Projects in the National Science \& Technology Pillar Program of China under Grant no. 2015ZX04005001.

\section{References}

[1] D.E. Dimla, P.M. Lister, On-line metal cutting tool condition monitoring. Force and vibration analyses, International Journal of Machine Tools and Manufacture 40 (2000) 739-768.

[2] S. Purushothaman, Y.G. Srinivasa, A back-propagation algorithm applied to tool wear monitoring, International Journal of Machine Tools and Manufacture 34 (1994) 625-631.

[3] I.N. Tansel, C. Mekdeci, C. Mclaughlin, Detection of tool failure in end milling with wavelet transformations and neural networks (WT-NN), International Journal of Machine Tools and Manufacture 35 (1995) 1137-1147.

[4] O. Masory, Detection of tool wear using multisensor readings defused by artificial neural network. International Society for Optics and Photonics1991, pp. 515-525.

[5] S.S. Rangwala, D. Dornfeld, Integration of sensors via neural networks for detection of tool wear states, Intelligent and Integrated Manufacturing Analysis and Synthesis (1987) 109-120.

[6] D.E. Dimla, Multivariate tool condition monitoring in a metal cutting operation using neural networks [University of Wolverhampton1998.

[7] S. Rangwala, D. Dornfeld, Sensor integration using neural networks for intelligent tool condition monitoring, Journal of Engineering for Industry 112 (1990) 219-228.

[8] K. Zhu, Y. San Wong, G.S. Hong, Wavelet analysis of sensor signals for tool condition monitoring: a review and some new results, International Journal of Machine Tools and Manufacture 49 (2009) 537-553.

[9] P.M. Lister, On-line measurement of tool wear. 1993. 